\title{
La peinture sur lave émaillée des façades des églises parisiennes du XIX siècle : une expérience de réintroduction de la polychromie
}

Enamelled lava painting on the facades of Parisian churches of the 19th century: an experiment to reintroduce polychromy

Josette Saint-Martin

\section{CpenEdition}

Journals

Édition électronique

URL : http://journals.openedition.org/cel/1820

DOI : $10.4000 /$ cel. 1820

ISSN : 2262-208X

Éditeur

École du Louvre

Référence électronique

Josette Saint-Martin, "La peinture sur lave émaillée des façades des églises parisiennes du xıx

siècle : une expérience de réintroduction de la polychromie ", Les Cahiers de l'École du Louvre [En ligne], 13 | 2019, mis en ligne le 17 juin 2019, consulté le 17 septembre 2019. URL : http:// journals.openedition.org/cel/1820 ; DOI : 10.4000/cel.1820

Ce document a été généré automatiquement le 17 septembre 2019.

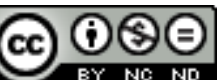

Les Cahiers de l'École du Louvre sont mis à disposition selon les termes de la licence Creative Commons Attribution - Pas d'Utilisation Commerciale - Pas de Modification 4.0 International. 


\section{La peinture sur lave émaillée des façades des églises parisiennes du XIX ${ }^{\mathrm{e}}$ siècle : une expérience de réintroduction de la polychromie}

Enamelled lava painting on the facades of Parisian churches of the 19th century: an experiment to reintroduce polychromy

Josette Saint-Martin

Les clients qui découvraient, jusqu'en 2005, Paris depuis la terrasse de La Samaritaine et tentaient de repérer les sites sur sa table d'orientation ${ }^{1}$, ignoraient probablement qu'elle était issue d'une technique née au XIX ${ }^{\mathrm{e}}$ siècle dans le $10^{\mathrm{e}}$ arrondissement de la capitale : celle de la peinture sur lave émaillée.

L'histoire de la technique de peinture sur lave émaillée est le fruit de la volonté d'un homme, Gilbert Joseph Gaspard, comte de Chabrol de Volvic ${ }^{2}$, préfet de Paris entre 1812 et 1830, qui souhaitait développer l'économie de sa région d'origine, Volvic. Cette histoire recouvre à la fois celle d'une innovation en matière de peinture, celle de la volonté de certains architectes comme Jacques Ignace Hittorff de réintroduire de la polychromie sur les façades des édifices et, enfin, celle du développement urbanistique et économique des nouveaux quartiers du nord de Paris.

2 Cette tentative de réintroduction de la polychromie sur les façades des églises parisiennes avec la peinture sur lave émaillée s'est déroulée entre 1827, date à laquelle Ferdinand-Henri Mortelècque, peintre et chimiste, a réussi à réaliser, avec cette technique, des peintures aux couleurs nuancées et aux qualités de résistance reconnues et, 1874, date à laquelle les tympans au-dessus des portes du porche de l'église SaintJoseph-des-Nations ${ }^{3}$ ont été ornés de peintures de Paul Balze ${ }^{4}$.

Pourtant, dès la fin du XIX ${ }^{e}$ siècle, cette tentative est tombée dans l'oubli, jusqu'à la repose des peintures sur lave émaillée de Jules Jollivet sur la façade de l'église SaintVincent-de-Paul ${ }^{5}$. La recherche conduite en 2017 a tenté d'apporter des éléments de 
réponse sur les raisons de cette indifférence qui s'est vérifiée jusque dans l'historiographie quasiment inexistante sur le sujet.

\section{Un procédé conçu et produit dans les $9^{\mathrm{e}}$ et $10^{\mathrm{e}}$ arrondissements de Paris}

Gilbert-Joseph-Gaspard de Chabrol fut l'un des premiers politiques à modifier l'aspect moyenâgeux du paysage parisien. En 1826, il utilisa la pierre d'origine volcanique de Volvic pour les bordures des trottoirs parisiens. Vers 1827, il souhaita faire émailler cette lave auvergnate pour l'employer dans la fabrication des plaques des rues de Paris ${ }^{6}$. Il encouragea également les beaux-arts et soutint de nombreuses commandes, en particulier dans les églises parisiennes en accordant des subventions aux fabriques. Très attaché à son Auvergne natale, Chabrol considérait la pierre de Volvic comme un matériau noble. C'est donc sous sa gouvernance que se conjuguent les destinées du Nord-Ouest parisien et d'une technique artistique nouvelle, la peinture sur lave émaillée pour laquelle il s'attacha à solliciter des chimistes et des artistes.

Dès 1828, alors que Chabrol voulait réhabiliter l'art du vitrail, il chargea Abel de Pujol, peintre en vogue, de diriger, dans le quartier de la Foire Saint-Laurent ${ }^{7}$, les peintres verriers anglais pour réaliser ses vitraux destinés à l'église Sainte-Élisabeth-deHongrie ${ }^{8}$. C'est aussi pour cette église que l'artiste reçu du préfet Chabrol, vers 1828, la commande d'un devant d'autel composé de trois médaillons: La Foi, La Charité et L'Espérance, peints sur lave émaillée ${ }^{9}$. Ce fut la seule œuvre réalisée sous l'administration du préfet Chabrol grâce aux recherches de Mortelècque. « M. Chabrol de Volvic, toujours attentif aux embellissements de la capitale, chargeait M. Abel de Pujol d'exécuter une peinture sur lave pour décorer le maitre-autel de l'église Sainte Élisabeth. Ce fut pour M. Mortelècque un jour de bonheur que celui-là, car tous les essais qu'il avait faits avaient été livrés au commerce ; ce n'était pas là son but, il visait plus haut, à la peinture monumentale. $»^{10}$ lit-on dans la presse de l'époque.

4 Ferdinand-Henri-Joseph Mortelècque ${ }^{11}$, artiste-artisan reconnu pour sa maîtrise dans la fabrication des couleurs pour porcelaine et pour verre, mit au point la technique de la peinture sur lave. Grâce au blanc d'émail, «le blanc Mortelècque », dont il couvrit les plaques de lave, on put exécuter sur des supports de grandes dimensions, dont la planéité était conservée à la cuisson, des peintures en couleurs vitrifiables réunissant éclat et solidité et, en plus, inaltérables.

Entre 1825 et 1827, Mortelècque réussit à sortir de ses fours, rue du Faubourg-SaintMartin, la première œuvre peinte sur lave émaillée, une Tête de vieillard. Entre 1832 et 1838 , l'entreprise de Mortelècque resta dans cette rue, s'installant dans différents ateliers. En 1824, les frères Bosson, négociants en vins, en plateaux de noyer et en blocs bruts de lave, installés à Volvic ${ }^{12}$, disposaient d'un terrain et d'un entrepôt situés 20 , quai Saint-Sabin sur le nouveau canal Saint-Martin, à proximité de l'atelier de Mortelècque, favorisant l'approvisionnement en bloc de laves qui étaient ensuite débités à Paris ${ }^{13}$. À la mort de Mortelècque, son atelier est repris par Dubois et Pierre Hachette, peintres sur verre. L'entreprise est toujours installée rue du Faubourg-SaintMartin. Elle possède six feux-fours et le volume de lave traité est de 5000 tonnes selon un bulletin d'exposant ${ }^{14}$ de la maison Hachette à l'Exposition des produits de l'industrie française de 1844. Hachette est le seul fabricant qui expose et mentionne son travail sur lave émaillée lors de cette manifestation. 


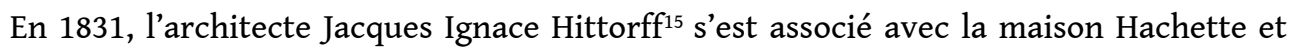
Cie. Il est ainsi devenu directeur de ce qui fut probablement la première entreprise d'émaillage sur lave. Elle était alors située au 40, rue Coquenard ${ }^{16}$, dans le $9^{\text {e }}$ arrondissement, après avoir probablement été expropriée du Faubourg-Saint-Martin du fait du passage du chemin de fer du Nord. Les six devants d'autel en lave émaillée réalisés sous la direction d'Hittorff pour l'église Notre-Dame-de-Lorette ${ }^{17}$, sont sortis des ateliers Hachette. Hittorff a l'ambition de former des artistes habiles et nombreux mais il démissionne des ateliers Hachette en 1838 pour éviter toute suspicion de conflit d'intérêts entre son travail d'architecte et son rôle de directeur de la manufacture. Son projet de formation resta lettre morte même s'il continua, néanmoins, à défendre ardemment la technique de la lave émaillée. En 1855, la maison Hachette s'installe 9, rue Fénelon, à côté de l'église Saint-Vincent-de-Paul, probablement dans l'espoir d'obtenir la commande pour les laves émaillées du porche de cette église. Un atelier et des fours spécialement construits pour ce chantier seront installés : "Car il faut savoir qu'avant la cuisson, la couleur sur lave est presque aussi sensible au toucher que le pastel: on comprend alors les précautions qu'exige le transport de panneaux dont chacun pèse au moins $120 \mathrm{~kg}$ ", explique Jules Jollivet, auteur des cartons des peintures sur lave de la façade de l'édifice. À la mort de Pierre Hachette, c'est François Gillet, chargé de la première peinture sur lave de Saint-Vincent-de-Paul, La Trinité, qui reprend la direction des ateliers. La façade de l'atelier Gillet, rue Fénelon ${ }^{18}$, qui date de 1874 , est entièrement décorée de laves émaillées retraçant l'histoire des arts du feu et représentant Mortelècque et Jollivet aux côtés de céramistes illustres comme Bernard de Palissy. Cette façade, véritable affiche publicitaire, place ainsi le procédé de peinture sur lave émaillée dans une tradition séculaire.

\section{Le retour de la polychromie architecturale sur les façades des églises parisiennes}

6 Le XIX ${ }^{\mathrm{e}}$ siècle est persuadé que l'architecture peut changer la société, et que l'art de bâtir commande toutes les autres formes d'art plastique. Architecture et arts décoratifs doivent transformer les mœurs, d'où l'importance des programmes décoratifs et les débats sur la polychromie architecturale influencés par les recherches archéologiques de l'époque. La redécouverte de la couleur en architecture est passée à la fois par celle de la polychromie dans l'architecture antique, dont Hittorff fut l'ardent défenseur en France, et par celle du patrimoine national du Moyen Âge avec les restaurations des édifices religieux et royaux. À partir de 1837, le chantier de restauration de la SainteChapelle de Paris et de ses sculptures monumentales va alimenter la réflexion sur la polychromie monumentale. Pour Viollet-le-Duc: «La peinture décorative grandit ou rapetisse un édifice, le rend clair ou sombre, en altère les proportions ou les fait valoir ; éloigne ou rapproche, occupe d'une manière agréable ou fatigue, divise ou rassemble, dissimule les défauts ou les exagère ${ }^{19}$. »

La fascination pour l'Orient, qui jouit d'un regain important dès le début du XIX siècle, a pu aussi contribuer à une évolution du regard porté sur l'architecture polychrome. Les azulejos des monuments espagnols et les faïences des mosquées du Caire exerçaient une certaine fascination, selon Paul Sédille ${ }^{20}$. L'Exposition universelle de Paris en 1867 a mis en valeur les essais des céramistes français et anglais. La polychromie monumentale fait peu à peu son retour avec le développement de nouveaux procédés. 
En 1851, Jacques Ignace Hittorff, a exposé sa théorie de la polychromie dans son ouvrage De l'architecture polychrome chez les Grecs, ou restitution complète du temple d'Empédocle dans l'acropole de Sélinonte. Il voulait mettre son intérêt archéologique au service de ses connaissances des techniques dans les constructions modernes et la polychromie en architecture était son principal centre d'intérêt. C'est cette approche qu'il mettra en application pour la construction de l'église Saint-Vincent-de-Paul dont il fut l'architecte, succédant à son beau-père Jean-Baptiste Lepère.

7 Les églises qui bénéficièrent au XIX ${ }^{\mathrm{e}}$ siècle de peintures sur lave émaillée sur leur façade se trouvent dans l'ouest et le nord-ouest de Paris, c'est-à-dire dans les nouveaux quartiers de la capitale. Proche des lieux de livraison des laves brutes et de leur traitement, leur localisation s'inscrit dans la modernisation de l'urbanisme parisien avec l'emploi de nouveaux procédés de construction et de décoration. Les églises SaintVincent-de-Paul, Saint-Laurent, situées dans le $10^{\mathrm{e}}$ arrondissement, Saint-Augustin dans le $8^{\mathrm{e}}$, l'église de la Sainte-Trinité dans le $9^{\mathrm{e}}$, et les églises Saint-Ambroise et SaintJoseph-des-Nations dans le $11^{\mathrm{e}}$, ont été construites selon un schéma qui, selon la classification de J.-M. Leniaud ${ }^{21}$, se caractérise par une "monumentalisation significative $d u$ dynamisme religieux» qui s'amorce vers 1830 . Cette monumentalisation se manifeste par une situation en léger surplomb des rues qui conduisent à l'édifice ; une visibilité, grâce à une implantation dans la perspective ou au croisement des nouvelles artères de circulation, et des rampes d'accès qui conduisent à un important porche.

L'église Saint-Vincent-de-Paul (fig. 1) est l'exemple même de cette théâtralisation et de la mise en pratique des conceptions architecturales et polychromiques d'Hittorff dont la manifestation la plus éclatante n'est autre que l'installation des peintures monumentales sur lave émaillée en façade. Elle est la première église qui reçut sous son porche une peinture sur lave : La Sainte Trinité (fig. 2), en 1846, réalisée par Jules Jollivet comme les six autres peintures qui suivront, en 1860. La façade de l'église étant laissée en simple maçonnerie apparente et avec des détails ornementaux limités, le décor peint sur lave émaillée en devient un des éléments essentiels. Sa visibilité, comme on peut encore le constater aujourd'hui, est facilitée par la disposition des colonnes du portique, sur trois rangs et en nombre décroissant et par la situation surélevée de l'édifice.

Fig. 1 et Fig. 2

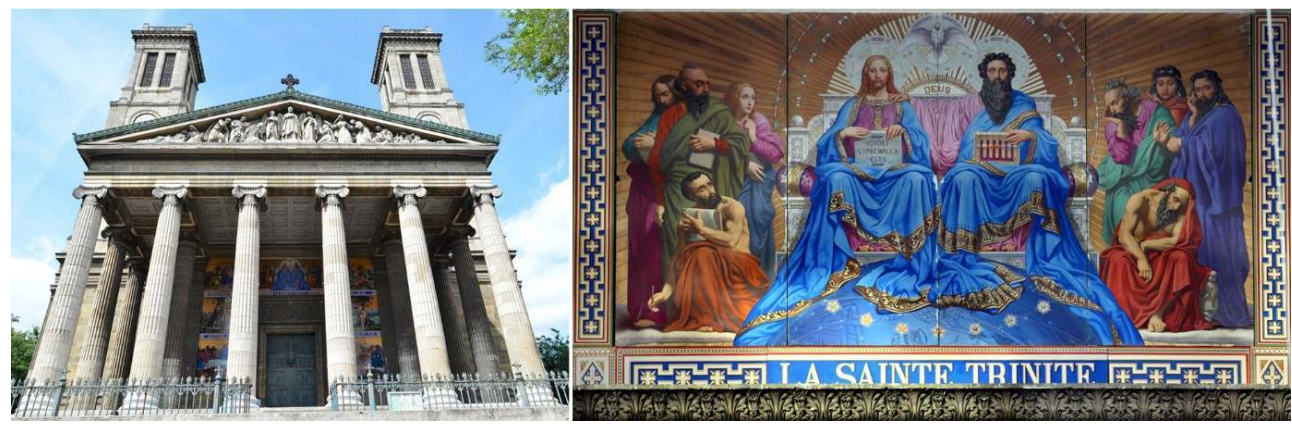

À gauche : Façade de l'église Saint-Vincent-de-Paul, 10e arrondissement, Paris. À droite : Le détail de la façade : La Trinité, première des peintures exécutées par Jules Jollivet en 1846 et composée de quatre panneaux.

(c) Ville de Paris - COARC - Claire Pignol ; @ B. Lodier 
Jollivet, peintre d'histoire, de genre et de nature morte, obtint du préfet Rambuteau la commande de La Sainte-Trinité pour le porche de l'église Saint-Vincent-de-Paul. Après des études d'architecture, il s'est tourné vers la peinture et est entré en 1822 à l'École des beaux-arts où il fut l'élève de Antoine-Jean Gros. C'est le hasard qui, selon ses écrits, lui fit rencontrer Hachette ${ }^{22}:$ «La vue de quelques peintures décoratives m'engagea à faire l'essai d'un procédé qui me semblait devoir ouvrir une nouvelle voie à l'art. C'était en 1844. L'épreuve ayant réussi, fut soumise sous les yeux du préfet de la Seine, qui décida qu'une peinture importante me serait commandée. M. Hittorff me proposa alors d'entreprendre l'exécution d'une immense décoration conçue autant pour fournir un exemple du principe qu'il avait savamment retrouvé, et qu'il soutenait ardemment, que pour faire une application concluante d'un procédé qui avait mérité sa confiance». Parmi les six tableaux qui accompagnèrent La Sainte-Trinitée $e^{23}$, trois figurent des épisodes de l'Ancien Testament: Création d'Ève, Péché d'Adam et Ève et Chute d'Adam et Ève; les trois autres figurent des épisodes du Nouveau Testament : l'Adoration des Mages, Baptême $d u$ Christ et La Cène. L'entreprise Hachette et Cie procéda à l'émaillage, comme le précisent les cartouches sous les peintures. Jollivet a semblé satisfait de la performance des fours puisque, lors de la réalisation du tableau de La Sainte-Trinité, il écrivit: "Aucun des accidents si fréquents dans la peinture en émail ne se manifesta. Soumis quatre fois au feu, le résultat fut toujours le même et l'on pût être ainsi assuré d'un succès constant de l'excellence de la combinaison des fours ${ }^{24}$. » Si l'on en croit toujours ses écrits ${ }^{25}$, il ne semble pas avoir rencontré plus de difficultés dans la réalisation des autres peintures. Jollivet évoque même une innovation relative à la conception des peintures ${ }^{26}$ de cette église : «Le tableau de Saint-Vincent de-Paule (sic) a été l'occasion d'un progrès important dans la peinture sur lave. [...]. Les plaques de laves ont été taillées carrément sans tenir compte des objets que traversaient les lignes droites des joints. Elles sont fixées aux parois du porche au moyen de vingt vis, logées dans des tampons de bronze scellés dans le massif de la construction. Cinq jours ont suffi pour établir les échafauds et tailler le mur, afin que le tableau ne fit pas saillie, et poser les quatre plaques et les bordures.»Ces informations, sur la pose des peintures qui ne doivent pas "faire saillie", confirment que les décors peints sont complètement intégrés à l'architecture de l'édifice.

8 Jollivet a écrit trois opuscules ${ }^{27}$ sur les peintures sur lave, en particulier sur celles de l'église Saint-Vincent-de-Paul. Dans celui de $1861^{28}$, il donnait des précisions quant au choix de l'iconographie et aux buts qu'il visait: «Il était au moins admissible que la partie extérieure d'une église, qui se présente tout d'abord aux regards, offrit aussi bien aux fidèles pour les affermir dans la foi, qu'aux indifférents pour les émouvoir, l'image des vérités qui sont à la base de notre sainte religion et la preuve de la divinité de sa révélation ». Il reprenait là l'approche qui semblait guider l'esthétique catholique de l'époque: enseigner et émouvoir. Il ajoutait s'être appuyé : "sur les premières instructions inscrites dans le catéchisme approuvé par l'archevêque de Paris en 1835, et telles furent les raisons qui dirigèrent le choix des sujets représentés sous le porche de Saint-Vincent ».

Sa description des peintures reflète cette volonté de concordance avec les attentes de l'Église catholique. Ses explications quant à l'iconographie retenue et sa signification sont très claires : « Au sommet l'image de la Très-Sainte-Trinité (sic), accompagnée des prophètes qui avaient annoncé les promesses de Dieu et de l'autre par les évangélistes qui en avaient constaté l'accomplissement. C'était le thème, le programme qu'il s'agissait de développer. Pour atteindre ce but, dans le panneau placé à la gauche du 
Père on devait représenter sur chacune des trois zones la création de l'homme, sa faute, sa punition ; dans le panneau de droite, c'est-à-dire du côté du Christ, en regard et dans le même ordre, l'avènement du sauveur accueillant les grands et les petits, son baptême qui efface la tache originelle et enfin la cène, premier acte de la rédemption ». Dans ses choix iconographiques, le peintre a donc fait preuve d'une maîtrise des textes chrétiens dans lesquels il a puisé de multiples références. Jollivet a trouvé son inspiration dans une tradition picturale qui remonte au Trecento de Giotto, comme dans sa représentation d'un saint Jean juvénile, yeux mi-clos ${ }^{29}$, mais il a pu tout autant s'inspirer d'une perception du Moyen Âge, revisitée par Viollet-le-Duc dans son choix d'un mobilier d'inspiration médiévale ${ }^{30}$. Il a probablement emprunté à Hittorff luimême les motifs polychromes des dosserets des bancs de pierres sur lesquels sont assis les anges regardant Adam et Ève chassés du paradis. Ils rappellent, en effet, les motifs géométriques et polychromes des devants d'autels, en peinture sur lave, conçus par l'architecte pour l'église Notre-Dame-de-Lorette. À cette diversité des sources s'ajoute, dans ses peintures, l'interprétation personnelle des textes par le peintre, qui adopte un style très narratif.

Une volonté de lisibilité très forte semble marquer ces peintures. Jollivet a, en ce sens, travaillé les effets de perspectives. Les peintures étant conçues pour être vues du sol, l'artiste avait créé une perspective qui était rendue par la taille des cartouches allant en grandissant au fur et à mesure de leur positionnement en hauteur. Une même logique décorative domine dans les peintures et leur cartouche. Elle se fonde sur quatre couleurs : le blanc, le bleu, le rouge et le vert. Le rendu de ces couleurs vives se trouve encore accentué par le support de lave, légèrement granuleux, qui accroche la lumière. L'impact des œuvres se trouve encore accru par les dimensions des figures, proches de la taille humaine. Visibles de loin, elles provoquèrent le scandale et leur retrait fut décidé dès 1861. Pourtant, dans le texte ${ }^{31}$ écrit par Jollivet après cet abandon, il précisait : «Mais sans redouter l'accusation de pruderie à laquelle m'auraient exposé des précautions trop affectées pour ne pas être ridicules, j'avais cependant cru pouvoir m'autoriser de quelques exemples pour emprunter aux vêtements flottants des anges, introduits dans le projet primitif, un moyen d'atténuer une nuditée ${ }^{32}$ que déjà devait affranchir de blâme la modestie du regard et de l'attitude de nos premiers parents. Mais conformément, sans doute, aux prescriptions du concile Quinisexte ${ }^{33}$ à l'égard de la fidélité qu'il faut observer dans la traduction du texte par la peinture, on me fit observer que l'Écriture n'avait pas signalé la présence des anges ou leur intervention dans les sujets de la création et de la désobéissance et qu'il y avait dans cette addition une sorte d'interprétation qu'il fallait éviter. Je me soumis au sacrifice d'un prudent artifice qui certainement eût soustrait ces compositions à des reproches. »C'est donc sur les injonctions du clergé lui-même que le peintre supprima les artifices visant à atténuer la nudité d'Ève (fig.3). C'est pourtant cette nudité qui s'offrait à toute personne passant à proximité de l'édifice que lui reprochèrent les paroissiens et les autorités ecclésiastiques. Selon l'article du père Bernard Horaist ${ }^{34}$, écrit en 2011 à l'occasion de la repose des laves émaillées sur la façade de l'église, le retrait des peintures, dès 1861, manifeste des limites de la concertation entre le clergé et les pouvoirs publics dans le suivi des commandes artistiques. Après en avoir référé au préfet de la Seine et à la Commission des beaux-arts, ordre est donné d'enlever les peintures, au prétexte que : «La vérité des formes, la splendeur du coloris blessent la décence » comme le relate un article dans Les Beaux-Arts ${ }^{35}$. Ce rejet de la part du clergé illustre les préoccupations de l'art chrétien du $\mathrm{XIX}^{\mathrm{e}}$ siècle qui devait, selon Isabelle 
Saint-Martin ${ }^{36}:$ «Se traduire par une esthétique, sinon par un style précis, et non par son sujet. [...] Un Christ, une Vierge, une scène biblique, ne suffisent pas à faire une œuvre chrétienne si l'inspiration de l'artiste ne l'est pas, si le traitement du sujet ne communique pas au spectateur cet état d'extase dans lequel doit le plonger la contemplation du beau spirituel. » Il semble bien, en effet, que le peintre ait mis une grande part d'interprétation et de créativité très personnelles à Saint-Vincent-de-Paul, ce travail étant probablement celui dans lequel il s'est le plus investi, comme le prouvent ses écrits.

Fig. 3

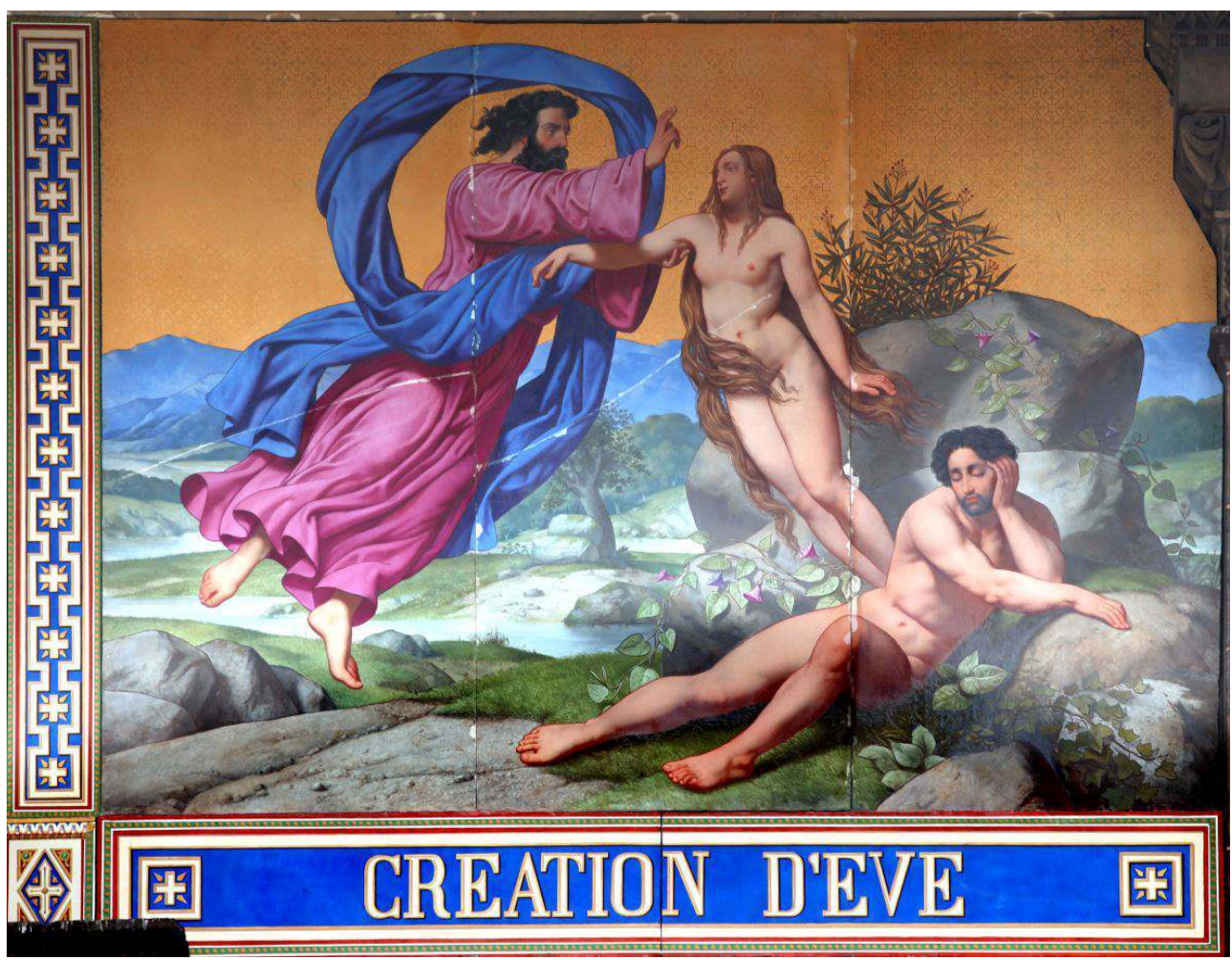

Création d'Eve, principal objet du scandale soulevé par les peintures de la façade de l'église SaintVincent-de-Paul, exécutée en trois panneaux sur lave émaillée par Jollivet, installée entre 1859-1860. Chaque panneau mesure environ 2,40 × 1,10 m.

(c) B. Lodier

Il faudra attendre 1998, et l'exposition à Paris ${ }^{37}$ « Lave émaillée : Un décor oublié du XIX ${ }^{e}$ siècle ", au Musée de la Vie romantique ${ }^{38}$, qui présentait La Sainte-Trinité, Adoration des Mages, Baptême du Christ, Création d'Ève et Adam et Ève chassés du paradis, pour que visiteurs, habitants du quartier et membres du clergé s'émeuvent et demandent le retour des peintures sur la façade de l'église Saint-Vincent-de-Paul. Dès 2009, La SainteTrinité retrouve sa place sous le porche de l'édifice tandis que les six autres peintures la rejoignent en $2011^{39}$.

Malgré ce retrait, la technique de la peinture sur lave émaillée, qui répondait bien au souci d'une polychromie extérieure résistant aux intempéries, continua à être employée, en particulier par Paul Balze, sur les façades d'autres églises parisiennes.

Les peintures sur lave émaillée de la façade de l'église Saint-Augustin, qu'il réalisa en 1865 s'inscrivaient dans une polychromie plus discrète que celles de Saint-Vincent-dePaul et ne soulevèrent aucun scandale. 
Dans ses choix architecturaux, Victor Baltard, chargé de la construction de l'édifice, alliait la modernité aux besoins spirituels en privilégiant les techniques architecturales et artistiques nouvelles ${ }^{40}$ dans un souci de rationalité. La formation initiale de peintre ${ }^{41}$ de Baltard contribuait certainement à l'intérêt qu'il portait à la décoration et c'est sans doute pour cela que la richesse décorative de l'intérieur se retrouve à l'extérieur et qu'il fit appel aux artistes les plus sollicités du Second Empire. La commande des peintures sur lave prévoyait que les cartons des peintures soient réalisés par Hippolyte Flandrin, Paul Balze se chargeant de leur exécution. Au décès de Flandrin, Balze fut mandaté pour réaliser les cartons et les peintures.

Paul Balze, peintre et copiste français, est issu d'une famille d'artistes originaire d'Arles. Il est admis à l'École des Beaux-Arts de Paris en 1831, puis, accompagné de son frère Raymond, il suit Ingres à Rome, où celui-ci vient d'être nommé à la tête de l'Académie de France. C'est à sa demande que les deux frères exécutent entre 1835 et 1847 , cinquante-deux copies $^{42}$ d'après les fresques des Loges et des Chambres du Vatican réalisées par Raphaël et son atelier au début $d u x^{e}{ }^{e}$ siècle. C'est d'ailleurs probablement lors de son séjour à Rome que Paul Balze fréquenta Hippolyte Flandrin et qu'il eut l'opportunité de rencontrer Victor Baltard. Balze s'intéressait à diverses techniques ${ }^{43}$ comme le montre la fresque du Couronnement de la Vierge réalisée entre 1859 et 1861 pour la voûte du chœur de l'église Saint-Symphorien de Versailles. Il fit plusieurs voyages en Italie et devant la dégradation des peintures murales des monuments italiens, il rechercha une technique qui résisterait aux attaques de l'environnement et du temps sans altérer la beauté des œuvres. Il s'intéressa particulièrement à la peinture sur émail pour la substituer à la fresque et offrir à la peinture murale un matériau résistant à ces attaques.

C'est à Rome, en 1862, qu'il réalisa ses premiers essais de peinture sur émail ${ }^{44}$ avec Le Triomphe de Galatée ${ }^{45}$ et Dieu le Père bénissant le monde après sa création, réalisés d'après une fresque de Raphaë ${ }^{46}$. En 1863, Ernest Vitet écrit dans la Gazette des Beaux-Arts, à propos de la Galatée présentée au Salon sous le numéro 1925 : «Comment s'y est-il pris, M. Balze, pour transporter sur de petits carreaux de cuisine un vrai Raphaël, pour nous rendre une fresque si classique avec un crayon tellement ferme, tellement correct, que son illustre maître (Ingres Ndr) s'en est réjoui ? Cette palette franche et fraîche (porcelainiers, émailleurs et verriers en perdent le sommeil), où donc a-t-il pris cela?» Ingres et Amédée Pichot ${ }^{47}$ auraient eu l'intention d'ouvrir un atelier de peinture sur lave émaillée que Paul Balze aurait dirigé. Il n'en fut rien et les procédés de Balze furent perdus ${ }^{48}$.

11 Même si la polychromie de la façade de l'église Saint-Augustin n'est pas aussi évidente que celle de Saint-Vincent-de-Paul, elle se retrouve par petites touches dans le rouge du dôme et des clochetons, le doré de la grande rose de la façade principale, les reflets cuivrés des portes et les peintures sur lave émaillée ${ }^{49}$. La façade est aussi animée par les nombreux jeux d'ombre et de lumière favorisés par un décor foisonnant de sculptures et de niches. Au-dessus des trois grandes portes du porche de l'église, les trois peintures sur lave émaillée de Balze sont placées dans des médaillons. Elles représentent les trois vertus théologales qui permettent à l'homme de vivre en communion avec Dieu: la Foi au centre, l'Espérance à droite de la précédente et la Charité à gauche.

Avec le thème de l'Église enseignante développé dans ses peintures, Balze les inscrit dans le programme iconographique global de la façade qui présente les Pères et les Docteurs de l'Église qui ont été la source de l'enseignement de l'institution catholique. 
Les peintures rappellent aux fidèles, dès leur arrivée au seuil de l'édifice, le cheminement qui s'offre à eux pour mériter la vie éternelle et la mission de l'Église dans cette démarche. Une approche qui ne pouvait que convenir aux ecclésiastiques. D'ailleurs, dans une lettre de Baltard ${ }^{50}$ à l'Archevêque de Paris, il explique qu'il a dû : "S'entendre avec M. le Curé de cette église [Saint-Augustin] pour le choix des sujets, peintures, sculptures et vitraux qui doivent entrer dans sa décoration.»

Dans ses Mémoires ${ }^{51}$, Haussmann, alors préfet de Paris, précise à propos de l'église de La Sainte-Trinité dont il a confié le projet à l'architecte Théodore Ballu ${ }^{52}$ : «M. Ballu suivant l'exemple de M. Baltard à Saint-Augustin, employa pour le même motif, la fonte et le fer dans la construction du chœur de La Trinité ». C'est probablement aussi le modèle de Saint-Augustin qui guida la décision de l'architecte de faire appel à Paul Balze pour réaliser des peintures sur lave émaillée sous le porche. Ce dernier était préoccupé par la technique, le coût des peintures et leur conservation. Il résolut les difficultés en employant des petits carreaux de terre de Ponchon ou de lave de dimensions égales, numérotés et parfaitement appareillés pour former un tableau quelle qu'en soit la taille. La Vision d'Ezéchiel, inspirée aussi d'un tableau de Raphaël ${ }^{53}$, a été réalisée avec son frère Raymond, selon cette technique d'une mosaïque de carreaux en lave émaillée. En 1891, cette mosaïque ${ }^{54}$ fut mise en place sur la façade de l'église Saint-Jacques de Montauban ${ }^{55}$.

13 C'est selon cette technique que Balze réalisa les peintures des tympans du porche de l'église de La Sainte-Trinité (fig. 4). Les peintures sont composées de carreaux de lave émaillée de dix centimètres de côté, technique ne nécessitant pas de grands fours.

La fonction éducative du décor de la façade pour illustrer le mystère de La Trinité, avec les ornements de son square et de sa façade, se retrouve dans les thèmes retenus par Balze pour les peintures sur lave émaillée. Au-dessus de la porte à droite de la porte centrale le message de la Pentecôte s'affiche: Accipite Spiritum sanctum (Recevez l'Esprit Saint). Depuis le $\mathrm{XI}^{\mathrm{e}}$ siècle, la Pentecôte est liée à La Trinité. Au-dessus de la porte gauche, le Christ figure frontalement assis sur une nuée, mains levées dans un geste de prise de parole, Ipsum audite (Écoutez-le). Au-dessus de la porte centrale, Dieu le Père est assis sur une nuée et montre Dieu le Fils assis auprès de lui et tenant un livre, Pater Judicium dedit Filio (Le Père donne sa justice au Fils). Entre le Père et le Fils, la colombe du Saint-Esprit complète La Trinité. Le Père et le Fils sont représentés frontalement, comme dans La Sainte-Trinité de Jollivet à l'église Saint-Vincent-de-Paul, mais la représentation de Balze est plus traditionnelle: le Père est un vieillard aux cheveux blancs, comme l'usage le veut depuis le $\mathrm{xv}^{\mathrm{e}}$ siècle, et il porte le globe terrestre. Si l'on en croit l'extrait du procès-verbal de la séance du 6 novembre 1867 de la Commission des beaux-arts, la réception de ces peintures fut bonne et les raisons de leur succès résident probablement, en partie, dans le choix de couleurs douces et dans l'iconographie consensuelle des sujets. 
Fig. 4

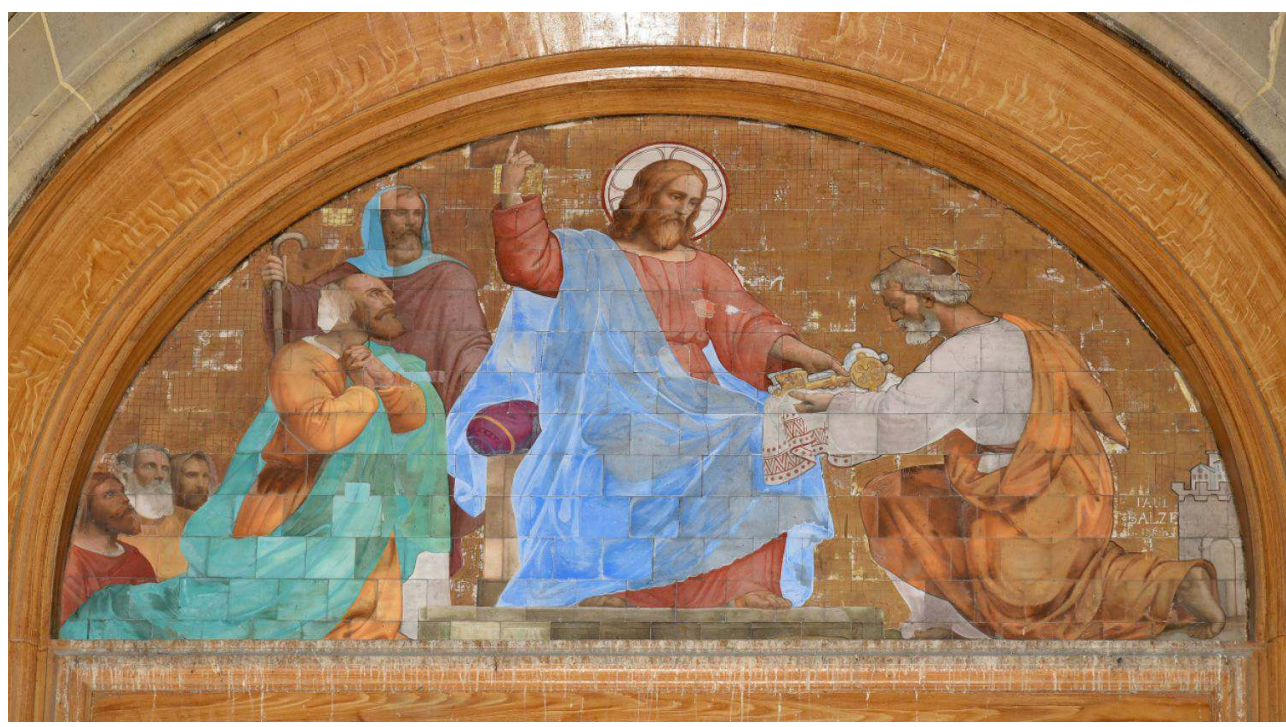

Accipite Spiritum sanctum : Recevez l'Esprit Saint, tympan de la porte droite du porche peinture exécutée par Paul Balze en 1867 avec la technique de petits carreaux de lave émaillée. Église de la SainteTrinité, $9^{e}$ arrondissement, Paris.

(c) Jean-Marc MOSER/COARC/Ville de Paris

En revanche, Balze rencontra plus de difficultés pour la réalisation des peintures sur lave de l'église Saint-Joseph-des-Nations dont il reçut commande par la Commission des Beaux-arts en 1876. Comme à Saint-Vincent-de-Paul, la vivacité des couleurs posa problème. La genèse des peintures fut difficile et la Commission fit considérablement modifier les représentations. Après un premier projet présentant saint Joseph, patron de cette église, entouré d'un groupe de laïcs et d'un groupe d'ecclésiastiques dont la composition fut refusée et pour lequel les couleurs étaient jugées trop violentes, c'est le même reproche que l'on fît aux autres peintures illustrant initialement Jésus au travail et La fuite en Égypte. Finalement, l'artiste réalisa des tableaux plus consensuels sur un fond d'or qui limitait les critiques sur la couleur. Le tympan central présente l'Apothéose de saint Joseph. Les anges placés sur les deux autres tympans symbolisent les vertus de saint Joseph : l'Ange de douceur et de chasteté sur le tympan de la porte à droite de la porte centrale, et l'Ange de vigilance et de fidélité au-dessus de la porte gauche.

C'est dans le contexte du réaménagement néo-gothique de la façade de l'église SaintLaurent mené entre 1862 et 1865 par l'architecte Claude Constant-Dufeux que Balze exécuta en 1870 les trois frises horizontales sur lave émaillée du nouveau tympan de l'église. La commande était une initiative de la Sous-commission des beaux-arts qui voulait une représentation à la gloire de saint Laurent, patron de l'église, réalisée « sur lave émaillée ou en mosaïque à fond d'or ${ }^{56}$ ". Ainsi les peintures sur lave étaient considérées comme une décoration en harmonie avec l'architecture quel qu'en soit le style, alors que neuf ans auparavant, les peintures sur lave de Saint-Vincent-de-Paul avaient été déposées. Il est vrai que la superficie couverte était moindre et le traitement iconographique plus conforme aux attentes du clergé. Là encore, elles s'inscrivent dans une façade qui n'oublie pas la polychromie. Celle-ci s'exprime aussi bien dans les cartouches peints et dorés aux armes de la ville de Paris placés sur les colonnettes qui surmontent deux petits contreforts situés aux angles de la partie 
centrale de la façade ${ }^{57}$ que dans les petits médaillons peints, au chiffre de saint Laurent, placés de part et d'autre de la baie ogivale de l'étage supérieur de la partie centrale.

Les peintures sont organisées en trois frises horizontales représentant le martyre et la glorification de saint Laurent. Dans la frise supérieure sont représentées la mort et l'apothéose du saint, tandis que dans la frise intermédiaire figurent saint Laurent et saint Sixte. Enfin, les trésors de l'église apparaissent dans la plus basse. Un bandeau sous chaque frise mentionne le nom des figures. Les esquisses très abouties de ces peintures se trouvent au Musée des Beaux-arts de la Ville de Paris. On y retrouve le fond doré préconisé par la Sous-commission des beaux-arts et mentionné dans l'extrait du procès-verbal de $1867^{58}$. Celui-ci donne à la peinture un style primitif assez évident qui n'a pas été retenu dans l'œuvre finale. Pour le matériau, un courrier de ConstantDufeux, daté du 9 mai $1869^{59}$ et adressé à « M. Hachette, fournisseur de laves émaillées, rue Doudeauville", nous apprend que Hachette devait fournir les laves à «la forme exacte de la surface à couvrir » ainsi que les échafaudages.

C'est dans la même configuration architecturale que celles des églises précédentes, sous des arcades du porche, que s'inscrivent les peintures sur lave émaillée réalisées à l'église Saint-Ambroise, en 1866, par Guiseppe Devers ${ }^{60}$ sur des cartons de Charles Soulacroix (fig. 5). Le sujet retenu est en relation directe et évidente, là aussi, avec le saint patron de l'édifice et sa légende. Saint Ambroise, évêque de Milan et Docteur de l'Église, est représenté sur le tympan au-dessus de la porte centrale, assis et revêtu de ses vêtements sacerdotaux violets. Eloquentia, allégorie de l'éloquence, qui le caractérisait, figure sur le tympan de la porte à droite du saint. Theologia, allégorie de la théologie à laquelle le saint a consacré une partie de son existence, est sur le tympan de la porte de gauche. Les trois figures sont représentées dans un cercle, entourées par des entrelacs formés de végétaux, proches de ceux des peintures décoratives de la nef de l'église Saint-Germain-des-Prés réalisées dans la première moitié du XIX siècle. Les trois figures, nommées et arborant leurs attributs clairement identifiables, sont l'unique sujet de ces peintures, sans autre figure à l'arrière-plan, dans un souci de simplicité mais aussi de lisibilité immédiate. La visibilité des œuvres est, là encore, favorisée par la netteté des contours et la vivacité des couleurs qui caractérise la peinture sur lave émaillée. 
Fig. 5

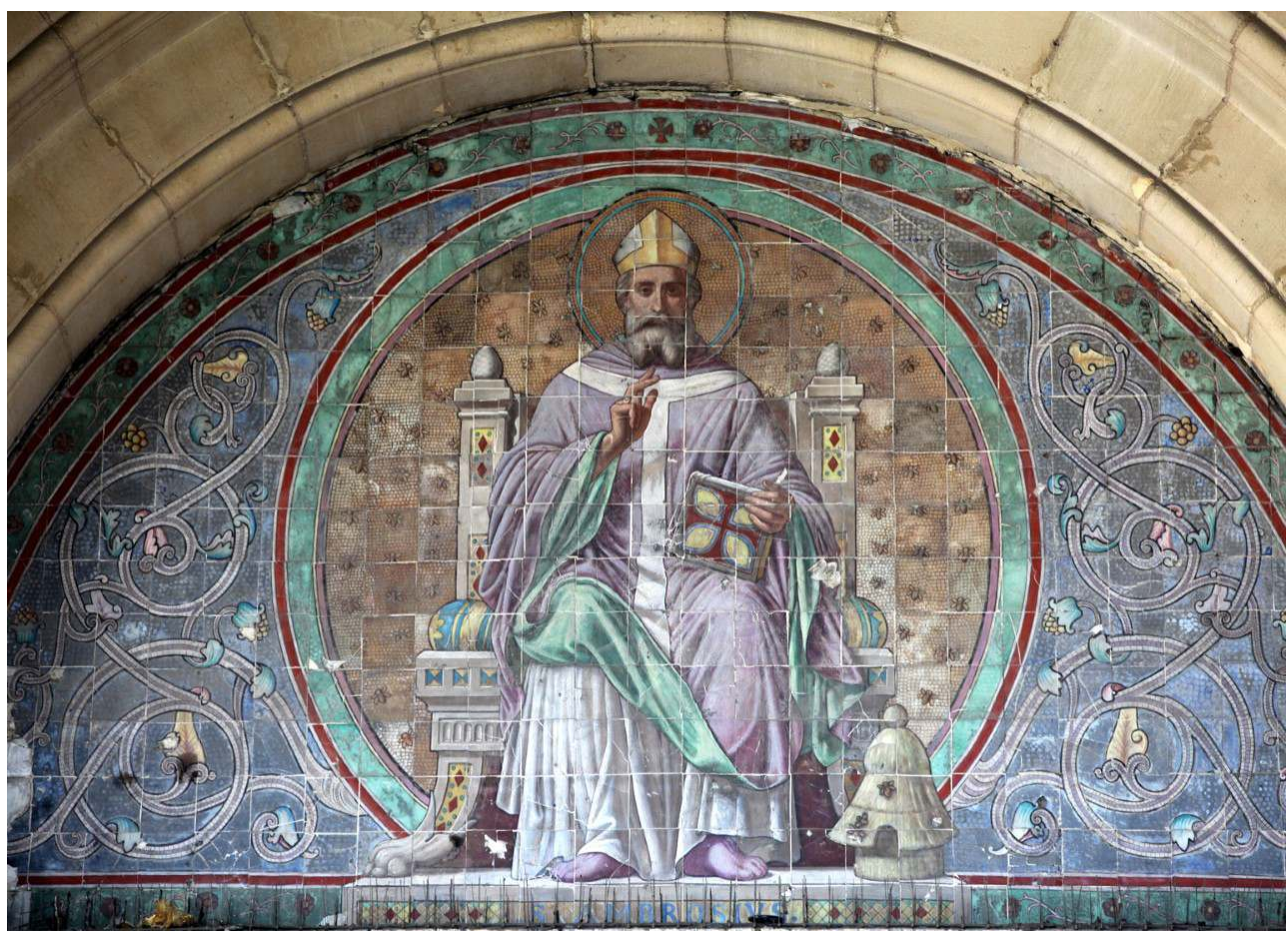

Saint Ambroise, peinture sur lave émaillée exécutée par Guiseppe Devers sur des cartons de Charles Soulacroix en 1866 pour le tympan de la porte centrale du porche de l'église Saint-Ambroise, $11^{\mathrm{e}}$ arrondissement, Paris.

(c) B. Lodier

\section{L'abandon d'une technique prometteuse}

Les études précédant les travaux de restauration sur les peintures sur lave émaillée des églises Saint-Vincent-de-Paul et Saint-Augustin que la COARC ${ }^{61}$ a entrepris dès 1998, montrent que la couche picturale est bien conservée, malgré un encrassement superficiel généralisé compte tenu de l'environnement auquel ces peintures sont soumises depuis plus d'un siècle. Le procédé de la peinture sur lave semble donc avoir eu toutes les qualités pour se substituer à la fresque, technique de l'art chrétien par excellence qui ne résistait pas à l'humidité du climat français. En 1839, le jury de la Commission des beaux-arts de l'Exposition des produits de l'industrie française prédit dans son rapport un grand avenir à la peinture sur lave : «Ce genre de peinture, si propre à toutes les décorations, à tous les tableaux, originaux ou copiés, qui doivent être placés dans les lieux humides, ou rester exposés à toutes les intempéries atmosphériques, ils auront très probablement l'inaltérabilité de la mosaïque faite en petits cubes d'émail, mais ils seront plus solides, et surtout infiniment meilleur marché que ce mode de peinture si long et si dispendieux. On peut, avec ce moyen, revêtir d'une peinture inaltérable les plus grandes surfaces et cacher mieux qu'en mosaïque les lignes de réunion ».

Pourtant, le succès ne fut pas au rendez-vous. Outre la difficulté d'approvisionnement en lave de Volvic d'une qualité correspondant à des fins artistiques relevée par Jollivet, la technique exigeait de la part des artistes une connaissance de l'évolution de la couleur des émaux après leur cuisson. Selon la complexité des tons, des valeurs et de la 
luminosité du dessin proposé par l'artiste sur le carton initial, plusieurs cuissons pouvaient être nécessaires et le travail de l'émail exige, encore aujourd'hui, une grande maitrise des teintes et de leur réaction à la cuisson. Outre Pujol, plusieurs artistes s'essayèrent à la technique de la peinture sur lave émaillée comme Victor Orsel et Alphonse Perrin, qui livrèrent des médaillons pour la cour intérieure des Beaux-Arts ${ }^{62}$, ou bien encore Pierre Étienne Perlet ${ }^{63}$ qui réalisa un grand buste du Christ pour l'église Saint-Nicolas-des-Champs ${ }^{64}$. Mais, tous se découragèrent face à la lenteur et à la minutie que cette technique exigeait.

Une autre cause probable de la désaffectation pour le procédé, peut être celle de la standardisation de l'architecture et de la décoration des nouvelles églises, liée au contrôle des coûts et de la qualité architecturale par l'État. Les peintures sur lave n'avaient pas leur place dans ce que Jean-Michel Leniaud ${ }^{65}$ nomme « le style utilitaire ", qui, à l'époque, trouva un écho dans l'évolution du goût. La sensibilité du milieu ecclésiastique à la thématique des œuvres, et à la moralité des artistes, a conduit au retrait des œuvres de Saint-Vincent-de-Paul aux motifs de décence et du caractère envahissant de la couleur. Une aspiration à la discrétion et à la modestie domine après les remous révolutionnaires. Les procédés industriels qui se développaient et facilitaient les reproductions standardisées d'images, donnaient par ailleurs à l'Église la maitrise de l'orthodoxie des images religieuses dès leur conception. Une imagerie "sulpicienne » était en train de naître, aux antipodes des peintures de Jollivet, mais correspondant davantage au goût de l'époque.

Cette courte page de l'histoire de l'art, en dépit de la modestie de la postérité de ces peintures, a ouvert la voie à la réintroduction de la couleur sur les façades. La polychromie s'installe peu à peu dans l'architecture des villes. Les éléments décoratifs de l'Art nouveau, comme au Castel Béranger ${ }^{66}$ ou sur les façades de La Samaritaine ${ }^{67}$, témoignent de cette réintroduction. Le choix du support pour cette polychromie connaîtra un continuel renouvellement, en rapport avec les évolutions techniques et les coûts de réalisation : fresque, lave, mosaïque.

Les questions que soulève aujourd'hui la restauration des peintures sur lave émaillée sont dues au manque de connaissance sur les procédés employés par les artistes qui pratiquèrent cette technique. Cette lacune prouve l'indifférence dont à elle a souffert pendant un siècle. Les restaurations des peintures sur lave des façades des églises parisiennes, commandées par la COARC, mais aussi celles des plaques ornées de décors floraux de l'ancien magasin de La Samaritaine semblent, cependant, témoigner d'un regain d'intérêt.

\section{NOTES}

1. L'illustrateur qui réalisa l'aquarelle à l'origine de la table d'orientation reste à ce jour inconnu. Elle fut réalisée vers 1923 par la maison Seurat installée à Saint-Martin près de Riom dans le Puyde-Dôme. Elle est conservée dans le projet de l'ensemble immobilier de LVMH et sera restaurée.

2. Né à Riom près de Volvic en 1773 et décédé à Paris en 1843. Ingénieur des ponts et chaussées. Il avait fait partie de l'expédition d'Égypte comme membre de la Commission des arts et sciences. 
3. Dans le $11^{\mathrm{e}}$ arrondissement.

4. Jean-Paul Balze (dit Paul Balze), né à Rome en 1815 et mort à Paris en 1884, peintre, élève d'Ingres.

5. Dans le $10^{\mathrm{e}}$ arrondissement.

6. Archives des découvertes et des inventions nouvelles, faites en 1831 et 1832, Paris, Treutel et Wurtz, 1833. Cette utilisation se répandit en France jusque dans les années 1950.

7. Sur l'emplacement actuel de la gare de l'Est.

8. Dans le $3^{\mathrm{e}}$ arrondissement.

9. Le devant d'autel est celui de la chapelle de la Vierge. Il est toujours en place.

10. Journal des Artistes du 26 janvier 1834.

11. Mortelèque ou Mortelècque, né à Tournai en 1774, mort à Paris en 1842.

12. Pierre Mondanel, «Les frères Brosson, deux agitateurs du Val d'Allier au XIX ${ }^{\mathrm{e}}$ ", L'Auvergne littéraire et artistique, $\mathrm{n}^{\text {os }}$ 204-205, Clermont-Ferrand, [s. éd.], 1970, p. 2-18.

13. Actuel 116, rue du Faubourg-Saint-Martin.

14. Service des collections documentaires de la Cité de la céramique, Sèvres, U 16, liasse 3, dossier 11 .

15. Né à Cologne en 1792, naturalisé français et formé à l'École des beaux-arts de Paris, mort en 1867. En 1840, il s'attache à l'aménagement de la place de la Concorde et de l'avenue des ChampsÉlysées. Il sera chargé de nombreuses constructions sous le Second Empire comme celles du cirque d'hiver (1851-1852) et de la gare du Nord (1861-1865).

16. Dans le $11^{\mathrm{e}}$ arrondissement.

17. Devant d'autel du maître-autel et ceux des autels particuliers qui sont toujours en place.

18. La maison existe toujours.

19. Eugène-Emmanuel Viollet-le-Duc, Dictionnaire raisonné de l'Architecture française, $18^{e}$ entretien, Paris, Morel, 1862, t. II, p. 336.

20. Paul Sédille, La céramique monumentale, conférence du 19 septembre 1878, Paris, Imprimerie nationale, 1879 , p. 97.

21. Jean-Michel Leniaud, La révolution des signes : l'art à l'église, 1830-1930, Paris, Éditions du cerf, 2007, p. 46-47.

22. Jules Jollivet, De la Peinture religieuse à l'extérieur des églises, à propos de l'enlèvement de la décoration extérieure du porche de Saint-Vincent-de-Paul, Paris, Imprimerie de A. Wittersheim, 1861, p. 49.

23. Les titres des peintures reprennent ceux donnés par l'artiste dans les cartouches qui accompagnent les peintures.

24. Jules Jollivet, « Nécrologie de M. Hachette », Revue générale d'Architecture et des Travaux publics, 1848, p. 352.

25. Jules Jollivet, De la Peinture religieuse à l'extérieur des églises, à propos de l'enlèvement de la décoration extérieure, op.cit. note 22, p. 48.

26. Jules Jollivet, « De la peinture sur lave », L'art en province, volume 11, Moulins, P. A. Desrosiers, 1850, p. 45- 49.

27. En 1861, ibid., en 1862, Peinture en émail sur lave. Sa raison d'être et de sa défense contre les obstacles opposés à son adoption, Paris, Wittersheim et, en 1867, De la polychromie de l'architecture par l'emploi des émaux, description d'un exemple à Deauville, Paris, Wittersheim.

28. Jules Jollivet, op. cit. note 27, p. 56.

29. Giotto, par exemple à la chapelle de l'Arena de Padoue. 
30. Eugène-Emmanuel Viollet-le-Duc, Dictionnaire raisonné du mobilier français de l'époque carlovingienne à la Renaissance, Paris, Gründ et Maguet, t. 1, 1873.

31. Jules Jollivet ; op. cit. note 22, p. 60.

32. Il s'agit de la nudité d'Ève.

33. Ou Concile de Trullo, réunit de 691 à 692 à l'initiative de l'empereur Justinien II. Il rassemble des évêques orientaux et veut mettre fin à la décadence des mœurs en réformant le droit canonique.

34. Bernard Horaist, 2011, COARC, boîte 3, Jules Jollivet.

35. Anonyme, 1861, Les Beaux-Arts : revue nouvelle, du 1er au 15 décembre 1861, Paris, t. 3 , p. 219.

36. Isabelle Saint-Martin, Regards chrétien-art sacré: regard du catholicisme sur l'art, France $\mathrm{XI}^{e}-\mathrm{XX}{ }^{e}$ siècles, Rennes, PUF, 2014, p. 52.

37. Paris, Musée de la Vie romantique, 15 octobre 1998-17 janvier 1999.

38. Cat. d'exp., Lave émaillée: Un décor oublié du XIXe siècle, sous la direction de Georges Brunel, Paris, Paris-Musées, 1998.

39. Toutes les peintures avaient été conservées à la COARC, Conservation des œuvres d'art religieuses et civiles de la Ville de Paris.

40. Georges Poisson, « L'église Saint-Augustin », Revue Napoléon III, Paris, $\mathrm{n}^{\circ}$ 10, 2010.

41. Il fut l'élève du peintre Guillaume Guillon Lethière (1760-1832).

42. Elles sont exposées à l'École nationale des beaux-arts de Paris.

43. Émile Bellier de la Chavignerie, Dictionnaire général des artistes de l'École française depuis l'origine des arts du dessin jusqu'à nos jours, Architectes, peintres, graveurs, sculpteurs et lithographes, Paris, Renouard, 1882, t. 1, p. 400.

44. Michel Baudat, «Deux arlésiens élèves d'Ingres, les frères Balze », La société des amis du vieil Arles, $\mathrm{n}^{\circ} 141$, juin 2009, p. 7 n. 15 (disponible en ligne).

45. Fresque de 1511-1514, pour le décor de la villa Farnesina à Rome.

46. Michel Baudat, 2009, op.cit. note 44, p. 8, n. 20.

47. Amédée Pichot (1795-1817), romancier, historien et traducteur français né à Arles.

48. Michel Baudat, op.cit. note 44, p. 3-32.

49. Les restaurations de la façade de l'église terminées en 2018 mettent en valeur cette légère polychromie.

50. Archives historique du diocèse de Paris, Carton en provenance de l'Archevêché, dossier Historique, «Rapport avec la Chancellerie », Lettre du 29 mai 1863.

51. Georges Haussmann, Mémoires du Baron Hausmann, Paris, Victor- Harvard, 1890, t. III, p. 547-548.

52. Né à Paris en 1817, il y est mort en 1885 , architecte en chef des travaux de la ville de Paris pour les édifices cultuels.

\section{Conservée à la Galerie Pitti.}

54. Le tableau comprend plus de 1000 carreaux de lave et Balze l'a agrandi $(4 \times 3 \mathrm{~m})$ par rapport à l'original qui ne mesure que quarante centimètres de haut.

55. Michel Baudat, 2009, op. cit. note 44, p. 9 n. 26.

56. Archives historiques de la Ville de Paris V. 32M 32-1

57. Clément de Ris, Ministère de l'Instruction publique, des cultes et des beaux-arts, Paris, 1876, t. 1, p. 51-52.

58. Archives historiques de la Ville de Paris, V 32 M32-1.

59. Ibid. 
60. Peintre et céramiste turinois (1823-11882) qui se lança dans le renouveau de la faïence architecturale, beau-frère de Charles Cordier, sculpteur partisan de la polychromie. Il réalisa vers 1851, un Christ en Majesté en peinture sur lave pour le tympan du porche de l'église Saint-Leu Saint-Gilles de Saint-Leu-la-Forêt dans l'Oise.

61. Conservation des CEuvres d'Art Religieuses et Civiles de la Ville de Paris.

62. Médaillons représentant Périclès, Auguste, Léon X et François $I^{\text {er }}$ exécutés par V. Orsel et A. Perin ; réalisés par la Société Hachette et cie, et installés rue Bonaparte entre 1838 et 1843.

63. Pierre Étienne Perlet dit Pétrus (1804-1843) ; peintre, dessinateur, graveur et lithographe. Sa tante, Aimée Perlet, est une des premières miniaturistes sur porcelaine du XIX ${ }^{\mathrm{e}}$ siècle. Né à Lyon, il vint à Paris pour intégrer l'atelier de Gros puis celui d'Ingres.

64. La peinture est toujours en place dans l'église.

65. Jean-Michel Leniaud, "La construction des églises du Concordat à la Séparation ", Quarantehuit / Quatorze, Paris RMN, 1995, nº 7, p. 11-17.

66. 14, rue la Fontaine, Paris $16^{\mathrm{e}}$, construit par Hector Guimard entre 1895 et 1898.

67. Construit entre 1905 et 1910.

\section{RÉSUMÉS}

L'expérience de réintroduction de la polychromie sur les façades des églises parisiennes du XIX siècle avec les peintures sur lave émaillée s'est déroulée, à Paris, entre 1827, date à laquelle Ferdinand-Henri-Joseph Mortelèque a mis au point les premiers essais réussis de peinture sur lave émaillée et, 1874, date à laquelle les tympans au-dessus des portes du porche de l'église Saint-Joseph-des-Nations dans le $11^{\mathrm{e}}$ arrondissement, ont été ornés de peintures sur lave émaillée peintes par Paul Balze. L'étude se concentre sur les six églises de Paris ayant reçu un décor peint sur lave émaillée en façade : Saint-Vincent-de-Paul, qui reste à ce jour le décor le plus connu par son importance et son histoire, La Sainte-Trinité, Saint-Augustin, Saint-Ambroise, Saint-Laurent et Saint-Joseph-des-Nations.

Des hypothèses sont avancées quant aux possibles raisons aussi bien techniques que concernant l'évolution du goût qui ont plongé cette tentative de réintroduction de la polychromie sur les façades des églises parisiennes du XIX ${ }^{\mathrm{e}}$ siècle avec la peinture sur lave dans l'oubli, jusqu'en 1998, année de l'exposition au Musée de la Vie romantique sur les laves émaillées de l'église SaintVincent-de-Paul.

The reintroduction of polychromy on the façades of nineteenth-century Parisian churches with paintings on enamelled lava took place, between 1827, the date on which Ferdinand-Henri-Joseph Mortelèque implement the first successful trials of painting on enamelled lava and, 1874, the date on which the tympana above the doors of the porch of the church of Saint-Joseph-des-Nations in the eleventh arrondissement were decorated by Paul Balze with paintings on enamelled lava. The study focuses on the six Paris churches with decoration painted on enamelled lava on the façade: Saint-Vincent-de-Paul, which to this day remains the best-known decoration in terms of its importance and history, La Sainte-Trinité, Saint-Augustin, Saint-Ambroise, Saint-Laurent and Saint-Joseph-des-Nations.

Hypotheses have been put forwards as to the possible reasons, both technical and with regards to changing taste, which plunged the attempt to reintroduce polychromy on the façades of 
nineteenth-century Parisian churches in obscurity, until 1998, the year of the exhibition at the Musée de la Vie Romantique of the enamelled lavas of the church of Saint-Vincent-de-Paul.

\section{INDEX}

Keywords : Nineteenth century, polychromy, churches, religious art, painting, lava, Paris, Paul Balze, Jules Jollivet, Giuseppe Devers

Mots-clés : XIXe siècle, polychromie, églises, art religieux, peinture, lave, Paris, Paul Balze, Jules Jollivet, Guiseppe Devers

\section{AUTEUR}

\section{JOSETTE SAINT-MARTIN}

Après une carrière à l'international dans un groupe pharmaceutique, Josette Saint-Martin depuis 2010 s'est tournée vers l'histoire de l'art. Après une licence, un I ${ }^{\text {er }}$ cycle à l'École du Louvre et les deux années de II ${ }^{\text {ème }}$ cycle, elle a clôturé sa deuxième année par un mémoire sur La peinture sur lave émaillée des façades des églises parisiennes $d u \mathrm{XI}^{e}$ siècle : une expérience de réintroduction de la polychromie. Elle poursuit actuellement une thèse qui porte sur les chemins de croix des églises parisiennes entre le $\mathrm{XIX}^{\mathrm{e}}$ siècle et Vatican II.

$* * *$

After an international career in a pharmaceutical group, Josette Saint-Martin turned to the history of art in 2010. After undergraduate and postgraduate studies at the École du Louvre, she wrote a dissertation on La peinture sur lave émaillée des façades des églises parisiennes $\mathrm{du} \mathrm{XI}^{e}$ siècle : une expérience de réintroduction de la polychromie. She is now writing a thesis on Ways of the Cross in Parisian churches between the nineteenth century and Vatican II. 